\title{
Descripción y seguimiento de pacientes COVID-19 en ventilación mecánica en unidades convertidas dirigidas por anestesiólogos: Clínica Alemana de Santiago
}

\author{
Description and monitoring of COVID-19 patients in mechanical ventilation in \\ converted units directed by anesthesiologists: Clínica Alemana de Santiago
}

Ya-Chuen Chuang 1,* (MD), Alexander Leisewitz V. ${ }^{1}$ (MD), Stefan Walkowiak N. ${ }^{1}$ (MD), Valentina Delgado R. ${ }^{1}$ (MD), Lucile Gignon² (MD, M.Sc.), Juan Abarca Z.2,3 (MD), Rodrigo Pérez³, Gabriel Cavada4 (Mg), Javiera Vargas Z. 2,4 (MD, Mg)

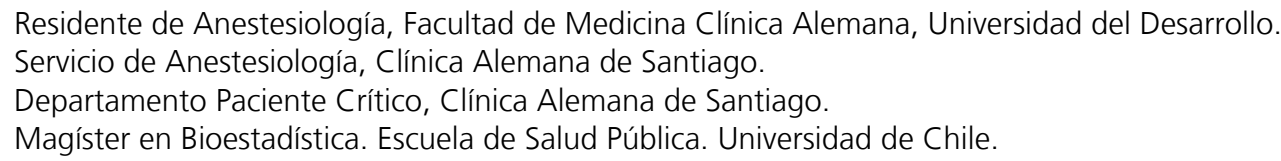

No hay fuentes de financiamiento.

No hay conflictos de interés.

Fecha de recepción: 12 de marzo de 2021 / Fecha de aceptación: 22 de marzo de 2021

\begin{abstract}
Introduction: The high demand for critical care beds and the need for mechanical ventilation generated by the pandemia of COVID-19 led the Ministry of Health of Chile to authorize both the conversion of beds and the use of anesthesia machines to mechanically ventilate COVID-19 patients. At Clínica Alemana de Santiago (CAS), these converted units were managed by nonintensivist anesthesiologists with technical support from the Intensive Care Unit (ICU). The objective of this study was to compare the survival rate of patients managed at converted and traditional ICU units. The secondary objective was to analyze the factors that may affect morbidity and mortality of these patients. Method: This prospective observational study included mechanically ventilated COVID-19 patients in both traditional and converted units. Patients were followed for 90 days starting at intubation day. Survival rate was determined at 30 and 90 days. A survival analysis was then performed. Results: 41 and 42 patients were admitted to CAS trough converted and traditional ICU units, respectively, between May 24 and June 30, 2020. There was not significant difference in survival rate between converted and traditional ICU units. Age (HR 1.1 per year) and SOFA (HR 1.4 per point) were associated with survival. Conclusions: At CAS, the survival of COVID-19 patients who required mechanical ventilation in converted units was not different as in those patients treated in traditional ICU units. Both age and SOFA were variables that can inform about prognosis of these patients.
\end{abstract}

Key words: Anesthesia, Critical care, COVID-19.

\section{RESUMEN}

Introducción: Dada la alta demanda de camas de paciente crítico y ventilación mecánica es que se autorizó el uso de máquinas de anestesia (MA) para ventilación mecánica prolongada y se ordenó la reconversión de camas para poder responder a la gran presión asistencial derivada de la pandemia por COVID-19 en nuestro país. Se investigó el curso clínico de pacientes COVID-19 ventilados por MA y ventiladores tradicionales en unidades convertidas manejadas por anestesiólogos en Clínica Alemana de Santiago (CAS) con la cooperación de Medicina Intensiva. Se comparó su sobrevida con respecto a pacientes COVID-19 venti-

*ychuang@alemana.cl

ORCID: https://orcid.org/0000-0003-4444-9662 
lados en Unidad de Paciente Crítico (UPC) tradicional y se estudiaron los factores que pudiesen contribuir al desenlace. Método: Se realizó un estudio observacional prospectivo donde se siguieron a los pacientes COVID-19, ventilados en UCI convertida y tradicional, desde su intubación hasta el día 30 y 90 para evaluar su sobrevida. Se registraron sus características basales, gravedad, morbilidad asociada, evolución ventilatoria y sistémica. Resultados: 41 pacientes ingresaron a unidades $\mathrm{UCI}$ convertidas entre el 24 de mayo al 30 de junio de 2020. No hubo diferencias significativas en la sobrevida con respecto a los pacientes que ingresaron a través de UCI tradicional. En nuestra muestra los factores asociados a sobrevida fueron edad con un $\mathrm{HR}=1,1$ (IC: $1,04-1,19)$ por año y SOFA con un $H R=1,4(I C: 1,19-1,65)$ por punto en el análisis multivariado ajustado. Conclusiones: No se observó diferencias estadísticamente significativas en la sobrevida de los pacientes que requirieron VM que ingresaron a través de $\mathrm{UCI}$ convertida o a UCI tradicional. Los factores asociados a sobrevida como edad y SOFA coinciden con la literatura previa, apoyando el uso de estos parámetros para orientar pronóstico.

Palabras clave: Anestesia, Unidades de Cuidado Intensivo, COVID-19.

\section{Introducción}

a pandemia de COVID-19 ha estresado todos los servicios de salud a nivel mundial.

- La letalidad derivada de las complicaciones respiratorias graves causadas por COVID-19 se ha visto asociada, en gran parte, por la necesidad simultánea de apoyo ventilatorio invasivo en muchos pacientes sobrepasando la capacidad del sistema de salud y su disponibilidad de equipos; esta situación significó la muerte del $75 \%$ de los pacientes en Wuhan, los cuales no recibieron ventilación mecánica en los inicios de la pandemia[1]. Otro ejemplo dramático es la cohorte de Lombardía con una mortalidad cercana al $50 \%$ de los pacientes que requirieron UCI durante la primera ola en Italia[2].

La FDA y el Ministerio de Salud chileno autorizaron el uso de máquinas de anestesia (MA) para ventilación mecánica (VM) prolongada dada la alta demanda de cuidados críticos derivados de la pandemia por COVID-19[3],[4],[5],[6],[7]. En este mismo contexto resultó imperativa la conversión de unidades médicas atendidas por médicos no intensivistas para poder manejar y tratar a estos pacientes[8],[9],[10].

La experiencia de VM prolongada con MA es muy escasa en el mundo, no encontrándose reportes del uso de estos dispositivos como proveedores de ventilación extendida previamente a esta pandemia, aunque los principios de la ventilación protectora se utilizan también durante una anestesia general[11], posicionando a los anestesiólogos y las MA como la segunda línea natural de respuesta ante la escasez de ventiladores mecánicos convencionales[12].

El objetivo es investigar el curso clínico de los pacientes COVID-19 ventilados por MA y ventiladores tradicionales ingresados en unidades convertidas manejadas por anestesiólogos, con el apoyo del equipo profesional de Unidad de Paciente Crítico (UPC) y comparar su sobrevida con los pacientes COVID-19 ingresados y ventilados en UPC tradicional como estándar de cuidado en nuestra institución y evaluar los factores que podrían haber contribuido al desenlace.

\section{Material y Métodos}

Se realizó un estudio observacional prospectivo, autorizado por la Unidad de Investigación de Clínica Alemana de Santiago (CAS), con exención de consentimiento informado aprobado por el Comité de Ética. Se resguardó la confidencialidad de la información sensible de los pacientes en todo momento.

Los criterios de inclusión fueron: Pacientes con edad mayor a 18 años, infección por COVID-19 demostrada por PCR (+) manejados en las unidades $\mathrm{UCI}$ convertidas manejadas por anestesiólogos en Clínica Alemana de Santiago. Los mismos criterios se aplicaron para los pacientes ingresados por UCI tradicional.

En nuestro centro se aumentaron las camas para manejo de paciente crítico al doble, con un total de 40 camas durante la primera ola en nuestro país; de estas 40 camas 12 pertenecían a las unidades convertidas dirigidas por anestesiología las que estaban organizadas 2 subunidades espacialmente separadas. El equipo de trabajo para cada subunidad contaba con 3 enfermeras, 3 técnicos en enfermería y 1 técnico de anestesia. En cada unidad se realizaba una visita diaria de jefatura técnica UPC, se contaba durante las $24 \mathrm{~h}$ con el apoyo de terapistas respiratorios para manejo de la ventilación y para la pronosupinación de los pacientes. En relación al uso de MA para ventilación prolongada, se contó con protocolos de revisión de las máquinas realizadas por técnicos de anestesia, chequeo similar al recomendado por la ASA[5]. Se procedía a chequear MA cada $72 \mathrm{~h}$, cal cada $24 \mathrm{~h}$ o antes si se encontraba virada, y filtros cada $12 \mathrm{~h}$, quedando registro de cada chequeo y del técnico responsable.

Se identificó, describió y siguió el total de pacientes que requirieron VM en las unidades convertidas para manejo de pacientes críticos para estudiar sus características basales, gravedad, morbilidad asociada, evolución ventilatoria y sistémica. Las variables registradas se encuentran en el Anexo 1.

\section{Anexo 1}

\section{Variables a registrar}

- Días de sobrevida

- Edad, sexo, IMC, gravedad por SOFA y APACHE

- Día de inicio de síntomas, día de hospitalización, día de intubación

- Antecedentes mórbidos

- Pafi, Gradiente Aa, GSA

- Necesidad de otros apoyos ventilatorios pre y post VM.

- Presencia de complicaciones :miocarditis, TEP, AKI, Infección bacteriana concomitante

- Necesidad de uso de bloqueadores neuromusculares, de Prono y ECMO 
Los registros se obtuvieron en forma diaria por visita presencial, revisión de ficha clínica electrónica (FCE) y registros de enfermería.

Se realizó seguimiento desde la intubación hasta el alta y, posteriormente, se realizó un seguimiento a 30 y 90 días para evaluar sobrevida.

Se obtuvo también la información de las características basales y de la sobrevida de los pacientes ventilados a través de ventiladores tradicionales durante el mismo período de tiempo en UPC convencional, para comparar con el estándar de cuidado.

Se describió la muestra a través de sus medidas de resumen (central y de dispersión) según la distribución de los datos. Se evaluó la diferencia entre variables continuas a través de una prueba de Mann-Whitney o t de student según correspondió y se evaluó asociación entre variables categóricas por test exacto de fisher.

Se realizó un análisis de sobrevida donde se estudió la asociación de las variables de estudio a través de un modelo de riesgos proporcionales de COX y también se comparó las curvas de sobrevida entre grupos a través de un test log-rank ajustando por Kaplan-Meier.S. Como análisis secundario se evaluó el poder de discriminación individual de variables asociadas a mortalidad a través del análisis del área bajo la curva de ROC (AUC).

Se utilizó una significación estadística del 5\% y los intervalos de confianza son de nivel 95\%.

Los datos fueron procesados en STATA versión 16.0.

\section{Resultados}

Durante el período de funcionamiento de las unidades de recuperación como $\mathrm{UCI}$ convertidas en CAS, tiempo comprendido entre el 24 de mayo al 30 de junio de 2020, ingresaron 41 pacientes que requirieron ventilación invasiva. Sus características basales se observan en la Tabla 1.

Dentro de los 41 pacientes, 33 eran hombres (81\%), 18 obesos (44\%), 18 tenían sobrepeso (44\%) y 16 hipertensión (39\%). El número promedio de días desde el inicio de los síntomas al ingreso hospitalario fue de 8,8 3,6 días y desde el inicio de los síntomas a la intubación fue de 11,3 4,3 días. El apoyo ventilatorio previo a la intubación se aplicó en 31 pacientes siendo el $68 \%$ a través de ventilación mecánica no invasiva y el $32 \%$ a través de cánulas de alto flujo. La PaFi promedio de in- greso fue de 144,4 48 y gradientes alveolo-arteriales de 289,4 119. Veintitres pacientes estuvieron en prono con BNM y solo uno se conectó a ECMO.

Del total de pacientes, 7 fueron ventilados exclusivamente por MA, 9 por ventiladores tradicionales y 25 fueron ventilados inicialmente por MA y, posteriormente, se les ventiló con ventiladores tradicionales observándose una mejoría leve en los parámetros sin ser estadísticamente significativo ( $\mathrm{PaFi}(\mathrm{p}=0,5)$ y $\left.\mathrm{pCO}_{2}(p=0,14)\right)$.

Diez pacientes fueron ventilados por MA por menos de 24 h los cuales tenían tendencia a tener peores PaFi en el primer día con medianas de 137 (p25-p75: 98-188) versus 150 p25p75 (117-188): 98-188), pero no significativamente distintas ( $p$ $=0,13$ ). Este grupo de ventilación por MA por menos de $24 \mathrm{~h}$ también presentó mayor $\mathrm{pCO}_{2}(p=0,04)$ que los que permanecieron ventilados por máquinas de anestesia, así como también mayores valores de Dímero $D(p=0,03)$, $P C R(<0,01)$ y leucocitos $(p=0,04)$.

Dentro de las complicaciones se observó: 3 miocarditis, 3 tromboembolismo pulmonar, 10 pacientes con daño renal agudo y 15 infecciones bacterianas.

En el mismo periodo ingresaron 42 pacientes en las unidades de cuidados intensivos tradicionales. Sus características basales se observan en la Tabla 1.

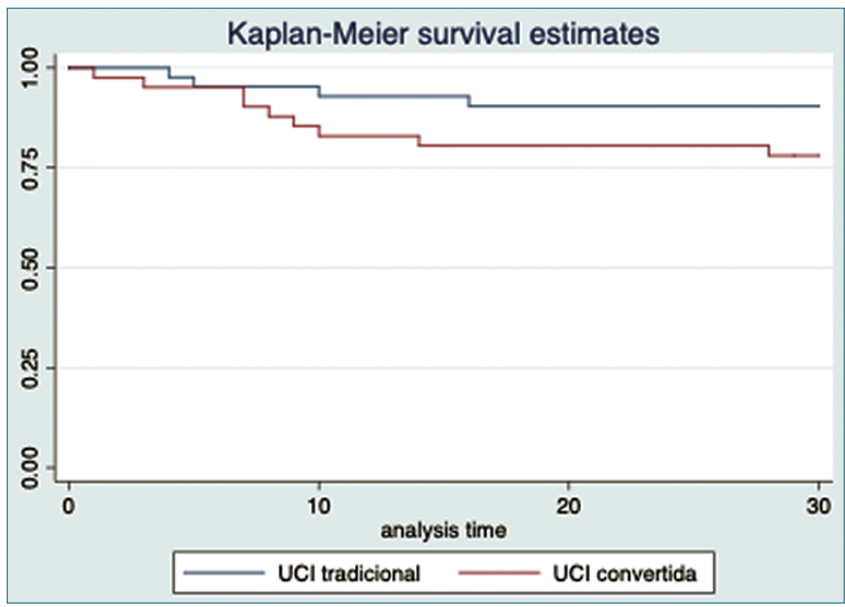

Figura 1. Curva de Kaplan-Meir para sobrevida de UCI tradicional vs UCl convertida.

\begin{tabular}{lccc}
\multicolumn{4}{c}{ Tabla 1. Características de pacientes ingresados a cuidados intensivos a través de UCl tradicional y UCl convertida } \\
\hline & UCl convertida & UCl tradicional & p-value \\
Edad (años) & $61,7-14$ & $60,9-9$ & 0,76 \\
Hombres & $81 \%$ & $79 \%$ & 0,82 \\
IMC & $29,6-4$ & $30,6-6$ & 0,39 \\
HTA & $39 \%$ & $64 \%$ & 0,021 \\
DM & $29 \%$ & $40 \%$ & 0,28 \\
PaFi & $144,4-48$ & $164,7-77,8$ & 0,37 \\
SOFA & $7-4$ & $4-2$ & $<0,01$ \\
APACHE & $11-4$ & $9-4$ & 0,04 \\
\hline
\end{tabular}


Los pacientes presentaron diferencias estadísticamente significativas en cuanto a la proporción de pacientes con hipertensión $(p=0,02)$, SOFA score $(p>0,01)$ y APACHE-II $(p=0,04)$, los detalles se observan en la Tabla 1.

La sobrevida de los pacientes que ingresaron a cuidados intensivos a través de las unidades convertidas vs las unidades tradicionales no fue estadísticamente significativa $(p=0,12)$, lo que se puede observar en la Figura 1.

Al evaluar los factores que estarían asociados con la sobrevida de los pacientes encontramos que la edad tiene un $\mathrm{HR}=$ 1,1 (IC: 1,05-1,18) por año, SOFA un $H R=1,4$ (IC: 1,15-1,64) por punto y APACHE-\| HR =1,18 (IC: 1,06-1,3) por punto. La significación estadística se mantiene para edad $(\mathrm{HR}=1,1$ (IC: 1,04-1,19) y SOFA HR = 1,4 (IC: 1,19-1,65) en el análisis multivariado ajustado por HTA. No fue significativa la asociación con la cantidad de días entre el inicio de los síntomas y la internación $(p=0,51)$, la cantidad de días entre el inicio de los síntomas y la intubación ( $p=0,63)$, ni tampoco la unidad de ingreso (convertida o tradicional) con un $p=0,14$.

Al evaluar la sobrevida de los 3 grupos de pacientes que fueron ventilados solo por máquinas de anestesia o solo por ventiladores tradicionales o en forma mixta no se observó diferencia estadísticamente significativa $(p=0,38)$ lo que se observa en la Figura 2.

Se observó un AUC para el poder de discriminación de la edad para el evento muerte de 0,84 (IC: $0,66-1,0)$ y para SOFA de $0,82(0,66-0,96)$.

El evento muerte se presentó en $21,9 \%$ de los pacientes, ocurriendo todas ellas dentro de los primeros 30 días, en los 60 días posteriores de seguimiento no se registraron muertes.

\section{Discusión}

En nuestro estudio observamos que no hubo diferencias significativas en cuanto a la sobrevida de los pacientes ventilados que ingresaron a cuidados intensivos a través de las unidades convertidas $o$ de las unidades tradicionales $(p=0,12)$. Las características generales de nuestros pacientes son muy similares a las descritas en otros reportes con predominio de pacientes con promedio de edad cercana a los 60 años, de sexo masculino, con sobrepeso u obesidad e hipertensión arterial[12]. Un elemento diferenciador de nuestra muestra fue la gran proporción de pacientes con VMNI previo a la intubación por sobre la cánula nasal de alto flujo (CNAF), dada la gran experiencia del equipo de kinesiología con este dispositivo, a pesar de las sugerencias de algunos grupos a favor de la CNAF[13].

La utilización de MA en forma prolongada mostró algunas limitaciones que quedaron en evidencia: dificultad para minimizar fugas en circuito, la incapacidad de medir $\mathrm{VCO}_{2}$ y la imposibilidad de dar humidificación activa sin condensar el circuito, limitaciones que también han sido reportadas por otros grupos[8]. Dado lo anterior, en nuestro centro se tomó la conducta con ciertos pacientes con evolución más tórpida, que apenas se liberaba un ventilador tradicional se intercambiaba con MA con la intención de mejorar los parámetros del paciente[8],[9],[12]. Esta conducta es concordante con los parámetros ventilatorios y de laboratorio que caracterizó al grupo de pacientes que fueron ventilados por menos de 24 h con máquinas de anestesia, pero los cambios en la PaFi y en $\mathrm{PCO}_{2}$ no fueron significati-

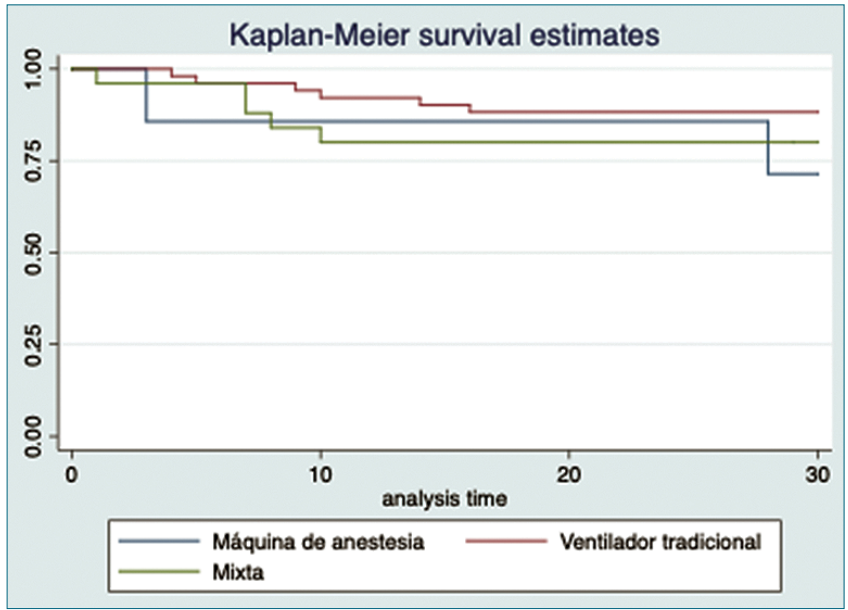

Figura 2. Curva de Kaplan-Meir para sobrevida de pacientes ventilados por MA, por ventilador tradicional o mixto.

vos en el primer control poscambio de dispositivo. Una posible explicación puede ser que la medición de los valores fue muy precoz en relación al cambio de máquina o de unidad.

Dentro de las experiencias internacionales, a la fecha de publicación, no se encontraron resultados de unidades reconvertidas con presencia de VM con máquinas de anestesia. Son varios los artículos que hacen referencia a los desafíos de trasladar la $\mathrm{UCl}$ a otras dependencias; respecto a espacio, staff, equipamiento, manejo de infecciones y bioseguridad[1],[8],[10],[15],[16], desafíos que también tuvieron que ser resueltos en nuestro centro.

Observamos que no hay diferencias significativas en la sobrevida de los pacientes que ingresaron a través de la UCI tradicional y convertida, esto probablemente gracias a la organización que se logró en las nuevas unidades, a la capacitación del personal de la unidad y a la unificación bajo una jefatura técnica acostumbrada al manejo de pacientes de UCI[17]. El manejo ventilatorio de los pacientes fue apoyado por el mismo equipo de terapistas respiratorios de la UPC de CAS, es decir, recibían una terapia kinésica homogénea. Cabe también mencionar que dentro del personal de enfermería y técnicos se contaba con funcionarios que provenían de la unidad coronaria, y en ciertos horarios también de pabellón, privilegiando que siempre hubiera personal más capacitado mezclado con personal más nuevo en cuanto al conocimiento de cuidados del paciente crítico con la idea de tener un apoyo permanente al personal menos experimentado.

Algunas revisiones sistemáticas y metaanálisis internacionales, que incluyeron pacientes de Asia, Europa y Norteamérica evidenciaron que a medida que avanzaba la pandemia, la mortalidad por COVID-19 reportada en UCI disminuyó: pasando de una mortalidad de más del 50\% a alrededor de 40\% desde marzo de 2020 hasta mayo de 2020. Esto probablemente debido al rápido aprendizaje sobre la enfermedad a nivel global, como también por un cambio en relación al criterio para ingreso a $\mathrm{UCl}$, por ejemplo, el uso de ventilación no invasiva fuera de UCI[18]. Otro estudio de S. Gupta et al. mostró una mortalidad de 35,4\% de pacientes COVID-19 hospitalizados en UCI, refiriéndose además que los factores asociados a mortalidad fueron edad, sexo masculino, obesidad mórbida, enfermedad 
coronaria, cáncer, disfunción orgánica aguda y admisión a centros con menos camas UCI[13]. Sin embargo, ninguno de los dos estudios previos explicita la mortalidad en UCIs convertidas de la forma en que lo muestra nuestro grupo, donde además la mortalidad fue menor.

Dentro de los factores asociados a sobrevida encontramos que tanto la edad como el puntaje SOFA fueron estadísticamente significativos, con un $H R=1,1$ (IC: $1,04-1,19)$ y $H R=$ 1,4 (IC: 1,19-1,65) respectivamente. El artículo de Ratschke et al. concluye que el SOFA no sería un buen predictor de mortalidad, siendo peor que la edad por sí solo como factor de riesgo, refiriendo entonces que no es un buen método para categorizar a los pacientes COVID-19 y determinar necesidad de ventilación mecánica[10]. Sin embargo, los outcomes medidos fueron distintos a los nuestros. En su artículo generan un outcome compuesto por porcentaje de mortalidad sumado a aquellos que egresaron a casas de acogida, mientras que nuestro grupo mide netamente mortalidad. Agregar además que en ese estudio para calcular el SOFA, utilizaron los peores valores obtenidos en las últimas 48 h previo al inicio de la ventilación mecánica, mientras que nosotros usamos el SOFA del día de la intubación[9]. En nuestro estudio el poder discriminación fue más alto para ambas variables, pero con un amplio intervalo de confianza probablemente asociado al tamaño muestral.

Al comparar la sobrevida de los pacientes ventilados sólo por MA, sólo por ventiladores tradicionales y los que fueron ventilados con ambos tipos de ventiladores, no se observó diferencia significativa, posicionando a las MA como herramienta válida de apoyo dentro del protocolo de nuestra UCI convertida y no como un equivalente a un ventilador tradicional dadas las limitaciones descritas con su uso prolongado. Creemos que el uso de MA debe ser racional, basado en la decisión clínica, con rotación con ventiladores tradicionales atendiendo la necesidad individual de cada paciente según su evolución y en nuestra cohorte esta conducta creemos pudo haber favorecido el hecho de no tener diferencias en la sobrevida encontrada.

Dentro de las principales limitaciones del estudio se encuentran: el bajo número de pacientes y el levantamiento de datos en un único centro. Sin embargo, estos corresponden a la totalidad de pacientes que ingresaron a UCI convertidas durante la primera ola en nuestro centro.

Cabe destacar que a pesar de realizar el estudio en un centro privado, este recibió pacientes derivados de otras instituciones (públicas y privadas) durante el período de investigación.

El presente artículo muestra la experiencia de nuestro centro tras la necesidad de conversión de camas en la primera ola de la pandemia por SARS-CoV-2 en nuestro país y no pretende extrapolar los resultados a otras instituciones.

Este estudio podría ser un primer paso para que en el futuro, pudiesen incorporar un mayor número de centros a nivel nacional para contrastar o refrendar la primera experiencia de reconversión de unidades en nuestro centro.

Agradecimientos: Extender nuestro agradecimiento a todo el personal que colaboró en el reto de transformar unidades: TENS, kinesiólogos, enfermeros/as, personal de apoyo y médicos anestesiólogos, los cuales se vieron expuestos a nuevas tareas y desafíos los cuales no estaban habituados. Destacar también el constante apoyo de todo el equipo de UPC para lograr unificar criterios y funcionamiento de todas las unidades.

\section{Referencias}

1. Goh KJ, Wong J, Tien J-CC, Ng SY, Duu Wen S, Phua GC, et al. Preparing your intensive care unit for the COVID-19 pandemic: practical considerations and strategies. Crit Care. 2020;24(1):215. Disponible en: https://doi.org/10.1186/s13054020-02916-4

2. Grasselli G, Greco M, Zanella A, Albano G, Antonelli M, Bellani G, Bonanomi E, Cabrini L, Carlesso E, Castelli G, Cattaneo S, Cereda D, Colombo S, Coluccello A, Crescini G, Forastieri Molinari A, Foti G, Fumagalli R, lotti GA, Langer T, Latronico N, Lorini FL, Mojoli F, Natalini G, Pessina CM, Ranieri VM, Rech R, Scudeller L, Rosano A, Storti E, Thompson BT, Tirani M, Villani PG, Pesenti A, Cecconi M; COVID-19 Lombardy ICU Network. Risk Factors Associated With Mortality Among Patients With COVID-19 in Intensive Care Units in Lombardy, Italy. JAMA Intern Med. 2020 Oct 1;180(10):1345-1355. Disponible en doi: 10.1001/jamainternmed.2020.3539.

3. Recomendaciones para el manejo de pacientes con COVID-19 con indicación terapéutica de ventilación mecánica que eventualmente son conectados a máquinas de anestesia." Rev Chil Anest 2020;49. Disponible en: https://doi.org/10.25237/revchilanestv49n03.09.

4. Commissioner of Food and Drugs - Food and Drug Administration Stephen M. Hahn M.D. Coronavirus (COVID-19) Update: FDA takes action to help increase U.S. supply of ventilators and respirators for protection of health care workers, patients. [Internet]. 2020 [citado 27 Marzo 2020]. Disponible en: https://www. fda.gov/news-events/press-announcements/coronavirus-covid19-update-fda-takes-action-help-increase-us-supply-ventilatorsand-respirators

5. Food and Drug Administration (FDA). Enforcement Policy for Ventilators and Accessories and Other Respiratory Devices During the Coronavirus Disease 2019 (COVID-19) Public Health Emergency [Internet]. 2020 [citado 22 Marzo 2020]. Disponible en: https:// www.fda.gov/media/136318/download

6. Anesthesia Patient Safety Foundation/American Society of Anesthesiologists. APSF/ASA Guidance on Purposing Anesthesia Machines as ICU Ventilators [Internet]. 2020 [Citado 7 Mayo 2020]. Disponible en: https://www.asahq.org/-/media/files/spotlight/anesthesia-machines-as-icu-ventilators-5-07.pdf?la=en\&has $h=164 A 428145$ ACF7B78E9F3732153C4953E3E8BE87

7. Ministerio de Salud de Chile. RESOLUCIÓN EXENTA No 158 Instruye sobre uso excepcional de máquinas de anestesia como ventilador mecánico para el manejo de pacientes con COVID-19 y aprueba protocolo de manejo clínico para estos efectos. [Internet]. 2020 [citado 2 Abril 2020]. Disponible en: https://www. sachile.cl/upfiles/userfile/files/MINSAL-Resoluci\%C3\%B3n-158. pdf

8. Xi AS, Chang MG, Bittner EA. Rapid establishment of an ICU using anesthesia ventilators during COVID-19 pandemic: lessons learned. Crit Care. 2020;24(1):388. Disponible en: https://doi. org/10.1186/s13054-020-03107-x

9. Jarzebowski M, Curran T, Dorsey M, Cederquist W, Claar D, Derrig $\mathrm{E}$, et al. Creating an intensive care unit from a postanesthesia care unit for the COVID-19 surge at the Veterans Affairs Ann Arbor Healthcare System. Fed Pract. 2020;37(8):348-53. Disponible en: https://doi.org/10.12788/fp.0020

10. Raschke RA, Agarwal S, Rangan P, Heise CW, Curry SC. Discriminant accuracy of the SOFA score for determining the probable 


\section{Artículo Original}

mortality of patients with COVID-19 pneumonia requiring mechanical ventilation. JAMA [Internet]. 2021; Disponible en: http:// dx.doi.org/10.1001/jama.2021.1545

11. Güldner A, Kiss T, Serpa Neto A, Hemmes SNT, Canet J, Spieth $\mathrm{PM}$, et al. Intraoperative protective mechanical ventilation for prevention of postoperative pulmonary complications: a comprehensive review of the role of tidal volume, positive end-expiratory pressure, and lung recruitment maneuvers. Anesthesiology. 2015;123(3):692-713. Disponible en: https://doi.org/10.1097/ ALN.0000000000000754

12. Peters AW, Chawla KS, Turnbull ZA. Transforming ORs into ICUs. N Engl J Med. 2020;382(19):e52. Disponible en: https://www. nejm.org/doi/full/10.1056/NEJMc2010853

13. Gupta S, Hayek SS, Wang W, Chan L, Mathews KS, Melamed $\mathrm{ML}$, et al. Factors associated with death in critically ill patients with Coronavirus disease 2019 in the US. JAMA Intern Med. 2020;180(11):1436. Disponible en: https://doi.org/10.1001/jamainternmed.2020.3596

14. Cinesi Gómez C., Peñuelas Rodríguez Ó., Luján Torné M., Egea Santaolalla C., Masa Jiménez J.F., García Fernández J. Clinical consensus recommendations regarding non-invasive respiratory support in the adult patient with acute respiratory failure se- condary to SARS-CoV-2 infection. Med Intensiva. 2020;502105691:30094-30102. doi: 10.1016/j.medin.2020.03.005

15. Williams MS, Zakaria S, D'Alessandro M, Kraeuter S, Hicks $\mathrm{H}$. Alternative cardiac intensive care unit locations during the COVID-19 pandemic at an academic medical center. Heart Lung. 2021;50(2):296-7. Disponible en: https://dx.doi. org/10.1016\%2Fj.hrtlng.2021.01.003

16. Carmona MJC, Quintão VC, Melo BF de, André RG, Kayano $\mathrm{RP}$, Malbouisson LMS, et al. Transforming operating rooms into intensive care units and the versatility of the physician anesthesiologist during the COVID-19 crisis. Clinics (Sao Paulo) [Internet]. 2020;75. Disponible en: http://dx.doi.org/10.6061/clinics/2020/ e2023

17. Mari GM, Crippa J, Casciaro F, Maggioni D. A 10-step guide to convert a surgical unit into a COVID-19 unit during the COVID-19 pandemic. Int J Surg. 2020;78:113-4. Disponible en: https://doi. org/10.1016/j.ijsu.2020.04.052

18. Armstrong RA, Kane AD, Cook TM. Outcomes from intensive care in patients with COVID-19: a systematic review and meta-analysis of observational studies. Anaesthesia. 2020;75(10):1340-9. Disponible en: https://doi.org/10.1111/ anae. 15201 